\title{
Confirmación de un modelo explicativo del estrés y de los síntomas psicosomáticos mediante ecuaciones estructurales
}

\author{
Mónica Teresa González Ramírez ${ }^{1}$ y René Landero Hernández ${ }^{1}$
}

Forma de citar

González Ramírez MT, Landero Hernández R. Confirmación de un modelo explicativo del estrés y de los síntomas psicosomáticos mediante ecuaciones estructurales. Rev Panam Salud Publica. 2008;23(1): 7-18.

RESUMEN Objetivo. Evaluar mediante ecuaciones estructurales un modelo del estrés que integra la explicación de los sintomas psicosomáticos y la teoría transaccional del estrés.

Métodos. Se seleccionó una muestra representativa, aleatoria y estratificada de estudiantes de la Facultad de Psicología de la Universidad Autónoma de Nuevo León, México. Los sujetos se seleccionaron a partir del listado de alumnos proporcionado por la facultad. Se utilizaron cuestionarios de autoevaluación con adecuadas propiedades psicométricas para valorar cada una de las variables incluidas en el modelo. Los resultados se analizaron con el programa AMOS 5.0, empleando el método de máxima verosimilitud. Asimismo, se compararon el modelo estructural con variables observables y un modelo con variables latentes endógenas.

Resultados. Los resultados obtenidos confirman parcialmente el modelo presentado y corroboran los efectos del estrés y el cansancio emocional sobre los síntomas psicosomáticos, y de la autoestima, la autoeficacia y el apoyo social sobre el estrés. El modelo con variables observables $\left(\chi^{2} / g l=2,87 ;\right.$ GFI (índice de bondad del ajuste) $=0,985$; AGFI (índice de bondad de ajuste corregido) =0,946; RMSEA (error de aproximación cuadrático medio) $=0,072 ;$ IFI (índice de ajuste incremental $)=0,982)$ tiene mejor ajuste que el modelo con variables latentes $\left(\chi^{2} / g l=\right.$ 3,74; $G F I=0,924 ; A G F I=0,876 ; R M S E A=0,09, I F I=0,927)$. En ambos casos, el ajuste es adecuado.

Conclusiones. El modelo presentado constituye el principal aporte de este trabajo. Se trata de un modelo explicativo de síntomas psicosomáticos, con buen ajuste, que explica el 24,3\% de la varianza al utilizar variables observables y el $39,4 \%$ al utilizar variables latentes.

Palabras clave Trastornos psicofisiológicos, estrés, México.

Se consideran trastornos psicosomáticos o psicofisiológicos las dolencias

\footnotetext{
Cuerpo Académico en Psicología Social, Facultad de Psicología, Universidad Autónoma de Nuevo León. La correspondencia debe dirigirse a Mónica Teresa González Ramírez, Universidad Autónoma de Nuevo León, Facultad de Psicología, Cuerpo Académico en Psicología Social, Mutualismo 110 Colonia Mitras Centro, Monterrey, Nuevo León, CP 64460, México; tel.: +52 (81) 8333 8233, fax: +52 (81) 8333 8222, correo electrónico: monygzz77@ yahoo.com
}

físicas cuya aparición, mantenimiento o agravamiento puede relacionarse con factores psicológicos (1). Al hablar de síntomas psicosomáticos nos referimos a las dolencias físicas para las que no se tiene la posibilidad de practicar un diagnóstico médico que las explique (2); estos síntomas pueden ser aislados y no constituir necesariamente un síndrome que coincida con el cuadro de una enfermedad.
La investigación actual sobre los trastornos psicosomáticos se basa en dos principios: multifactorial y multidisciplinar (3). Dentro de esta perspectiva, se han realizado estudios considerando diferentes variables para explicar este tipo de trastornos, como por ejemplo, trabajos que utilizan la regresión logística (4-7) y demuestran que los trastornos y síntomas psicosomáticos pueden relacionarse con múl- 
FIGURA 1. Modelo explicativo del estrés y de los síntomas psicosomáticos

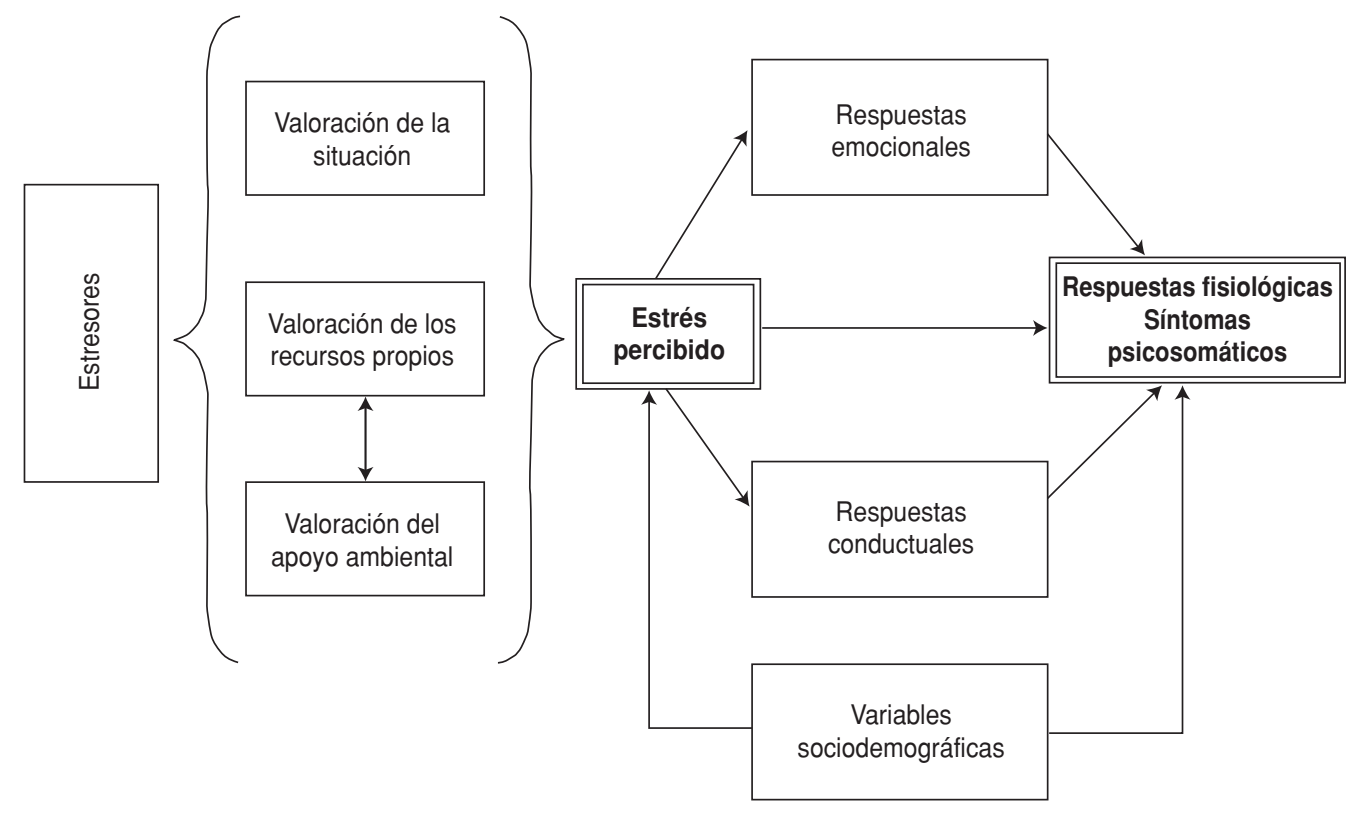

tiples factores. Entre estos factores se encuentran el estrés (5, 7-11), la depresión $(6,12,13)$, los trastornos de ansiedad $(4,13)$ y de personalidad $(9,14$, $15)$, el sexo (femenino) $(6,16,17)$, las formas de afrontamiento (10), la autoeficacia (7), el apoyo social (10), la autoestima (10) y la alexitimia (12).

La teoría transaccional del estrés de Lazarus y Folkman (18) proporciona un marco de referencia adecuado para la integración de los resultados de investigaciones que buscan explicar los síntomas psicosomáticos (2). Así, aunque existen diferentes aproximaciones al estudio del estrés, éste puede considerarse como: 1) una respuesta del individuo (activación simpática, liberación de catecolaminas o cortisol, ansiedad, ira, etcétera); en este caso el estrés actúa como variable dependiente; 2) un fenómeno del entorno externo (estimulación dolorosa, ruido, discusiones, etcétera); en este caso el estrés se considera variable independiente; y 3) una interacción (transacción) entre el individuo y el medio (como proceso) (19). Retomando la teoría transaccional, se puede considerar que el estrés no es exclusivamente un evento externo, del cual el sujeto es víctima; por el contrario, el estrés psi- cológico se define como una relación particular entre el individuo y su entorno, que él mismo evalúa como amenazante o desbordante respecto de sus recursos y que pone en peligro su bienestar (18). Dentro de la perspectiva transaccional del estrés, la evaluación cognitiva representa un aspecto central en la explicación del estrés; la evaluación es un proceso universal mediante el cual las personas valoran constantemente la significación de lo que ocurre en relación con su bienestar personal. La teoría de Lazarus y Folkman distingue tres tipos de evaluación: primaria, secundaria y reevaluación (20). La evaluación primaria se produce en cada transacción (o encuentro) con algún tipo de demanda externa o interna, y puede dar lugar a cuatro modalidades de evaluación: amenaza, daño o pérdida, desafío y beneficio; esta última no induce reacciones de estrés. La evaluación secundaria se refiere a la evaluación de los propios recursos para afrontar la situación. La reevaluación implica una retroalimentación y permite realizar correcciones respecto a valoraciones anteriores (20).

Considerando lo anterior y teniendo en cuenta las aportaciones de Lazarus y Folkman —referencia ineludible en el ámbito del estrés- (21), propusimos (6) un modelo explicativo del estrés y de los síntomas psicosomáticos basado en la teoría transaccional del estrés y en el modelo procesual del estrés de Sandín $(19,20)$ (figura 1). Este modelo, en el que se hace énfasis en los síntomas, postula que en presencia de estresores, una persona puede o no percibir estrés, en función de la valoración que hace el individuo de sí mismo (autoestima y autoeficacia), del apoyo social que percibe y de la valoración de la situación (como amenazante, desafiante o de daño o pérdida). Además, en este modelo se considera que las variables sociodemográficas pueden estar asociadas a la percepción de estrés y a los síntomas psicosomáticos. Cuando el sujeto percibe algún grado de estrés se pueden producir respuestas emocionales, conductuales y fisiológicas (síntomas psicosomáticos, tales como dolor de estómago, espalda, brazos, piernas, cabeza o pecho, vértigos, falta de aire, estreñimiento e indigestión). Asimismo, se plantea la posibilidad de que las respuestas emocionales y conductuales tengan algún efecto sobre los síntomas, ya que estas relaciones se han comprobado en otros estudios (2). 
El objetivo del presente estudio fue evaluar el modelo explicativo del estrés y de los síntomas psicosomáticos propuesto mediante ecuaciones estructurales.

\section{MATERIALES Y MÉTODOS}

\section{Participantes}

El estudio se realizó en 2005, según un diseño transversal. Se seleccionó una muestra representativa de estudiantes de la Facultad de Psicología de la Universidad Autónoma de Nuevo León, cuya población en ese momento era de 2410 estudiantes. El muestreo fue aleatorio estratificado y se realizó a partir del listado de alumnos proporcionado por la facultad, según la proporción de estudiantes por turno y sexo. El tamaño muestral se determinó con el programa nQuery Advisor.

Se decidió estudiar una muestra de estudiantes por considerarse que la prevalencia de síntomas en esta población es similar a la de otras poblaciones. En un estudio realizado en Alemania, Brasil, Chile, China, Estados Unidos, Francia, Grecia, India, Italia, Japón, Nigeria, Países Bajos, Reino Unido y Turquía (4), se encontró una presencia de síntomas en 19,7\% de las personas evaluadas (media entre países). En Alemania, la tasa de incidencia encontrada respecto de cualquiera de los diagnósticos somatomorfos fue de $25,7 \%$ (6). En un estudio realizado con estudiantes universitarios (22), se refirió que alrededor de $20 \%$ de los participantes presentaba de manera transitoria trastornos psicosomáticos. Por último, en un estudio realizado en México (8), se encontraron síntomas psicosomáticos en $21,8 \%$ de los estudiantes evaluados. Por lo tanto, sin dejar de lado las limitaciones relacionadas con la muestra, en este estudio se decidió evaluar el modelo propuesto en una muestra de estudiantes universitarios.

Participaron en el estudio 365 estudiantes, lo que representa 93,8\% del tamaño muestral predeterminado. En el cuadro 1 se presenta la distribución de los sujetos de la muestra estudiada por

CUADRO 1. Distribución por estratos (turno y sexo) de la población estudiada de alumnos de la Facultad de Psicología de la Universidad Autónoma de Nuevo León, México, 2005

\begin{tabular}{|c|c|c|c|c|c|}
\hline Estrato & Turno & Sexo & $\begin{array}{c}\text { No. total } \\
\text { de alumnos }\end{array}$ & $\begin{array}{l}\text { No. de alumnos } \\
\text { seleccionados } \\
\text { para el estudio }\end{array}$ & $\begin{array}{c}\text { No. de alumnos } \\
\text { participantes } \\
\text { en el estudio }\end{array}$ \\
\hline 1 & Matutino & Varones & 226 & 37 & 35 \\
\hline 2 & Matutino & Mujeres & 858 & 138 & 133 \\
\hline 3 & Vespertino & Varones & 90 & 15 & 13 \\
\hline 4 & Vespertino & Mujeres & 224 & 36 & 23 \\
\hline 5 & Nocturno & Varones & 169 & 28 & 27 \\
\hline 6 & Nocturno & Mujeres & 843 & 135 & 134 \\
\hline Total & & & 2410 & 389 & 365 \\
\hline
\end{tabular}

turno y sexo (estratos); 20,5\% era de sexo masculino y $79,5 \%$, de sexo femenino; proporciones que correspondían casi exactamente a las de la población de la facultad $(20,1 \%$ y $79,9 \%$ respectivamente, según los datos proporcionados por el Departamento escolar de la facultad). La edad media era 20,48 años (desviación estándar: 3,62 años) y la mediana, 20 años.

\section{Procedimiento}

Durante el mes de septiembre de 2005 , se citó a los estudiantes previamente seleccionados a una reunión - en sesiones programadas para cada turno-, se explicó a los asistentes que habían sido seleccionados aleatoriamente para representar a su facultad en una investigación y se aplicaron los instrumentos a aquellos que aceptaron participar. Posteriormente se buscó en las aulas a los aproximadamente 80 estudiantes que no habían asistido a las reuniones y se procedió con ellos de la misma manera que con los estudiantes que habían participado en dichas reuniones.

Para contrastar el modelo y cumplir con el objetivo del estudio, se midieron variables pertenecientes a cada elemento del modelo, con excepción de los estresores. En concordancia con esto, tampoco se midió la valoración que cada sujeto hacía de la situación estresante. Por lo tanto, el presente estudio es parcial. En cuanto a los recursos propios o personales, se evaluaron la autoestima y la autoeficacia. Para valorar el apoyo, se evaluó el apoyo social percibido. Como respuestas emocionales se evaluaron la depresión, la ansiedad y el cansancio emocional, y como respuestas conductuales, el afrontamiento. En la figura 2 se representa el modelo de partida.

\section{Instrumentos de evaluación}

Para la selección de los instrumentos utilizados se consideró su adecuación teórica con el estudio y los datos de validez y confiabilidad. Estos instrumentos fueron:

a) Síntomas psicosomáticos: se utilizó el cuestionario sobre la salud del paciente (Patient Health Questionnaire, PHQ), diseñado para medir la intensidad o gravedad de los síntomas somáticos a través de 15 ítems, con una consistencia interna satisfactoria (coeficiente $\alpha$ de Cronbach $=0,80)(23)$. Este cuestionario se ha aplicado en diferentes países (24). En un estudio donde se analizaron sus propiedades psicométricas en una muestra que incluía hispanos y no hispanos $(n=172)$ con altos niveles de síntomas medicamente inexplicables, se encontró el mismo grado de consistencia interna en ambos grupos (coeficiente $\alpha$ de Cronbach $=0,79$ ). Sin embargo, los autores refirieron diferencias en la validez; en los hispanos el PHQ tuvo una relación menor con los sín- 
FIGURA 2. Modelo inicial: modelo teórico para explicar el estrés y los síntomas psicosomáticos

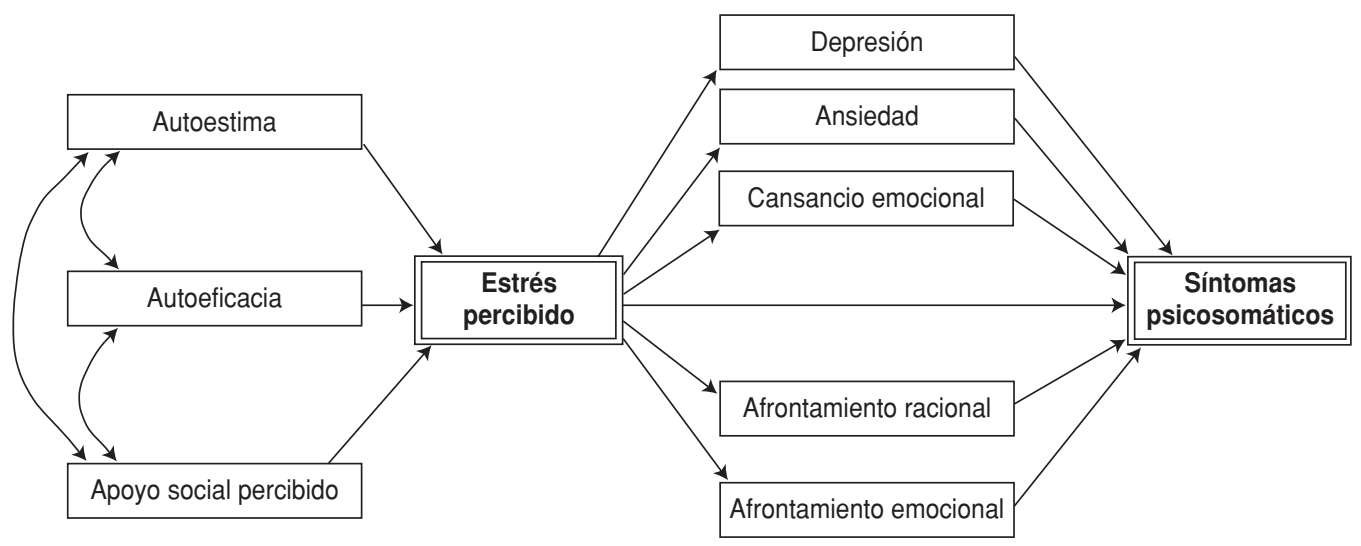

tomas medicamente inexplicables y mayor con el distrés (24). En México, este cuestionario ya se ha aplicado, con un coeficiente $\alpha$ de Cronbach de 0,77 (8).

b) Estrés: se utilizó la escala de estrés percibido (PSS) de 14 ítems (25), en la versión adaptada culturalmente a México (26), que presentó una consistencia interna adecuada (coeficiente $\alpha$ de Cronbach $=0,83$ ).

c) Depresión: como se puede presentar circularidad explícita cuando se produce una redundancia de ítems entre las medidas predictoras y de criterio (27), se consideró necesario contar con una medida de depresión que no fuera redundante con los síntomas psicosomáticos; así, se utilizó el inventario de depresión de Beck (28), que considera prioritariamente el componente cognitivo y consta de 21 ítems. El coeficiente $\alpha$ de Cronbach de esta escala osciló entre 0,76 y 0,95 en diferentes estudios (28).

d) Ansiedad: Inglés et al. (29) compararon los datos de confiabilidad y validez de diversas escalas; considerando sus resultados, además del número de ítems, se utilizó la escala de ansiedad social en adolescentes (Social Anxiety Scale for Adolescents, SAS-A), que consta de 18 ítems (30). El valor del coeficiente $\alpha$ de Cronbach notificado para esta escala fue de 0,91 (31).

e) Cansancio emocional: hace unos años se comenzó a hablar de "estudiantes quemados", en alusión al síndrome de desgaste (en inglés, burnout) producido por los estudios universitarios durante los últimos cursos de la carrera y el posgrado, que sólo se manifiesta como cansancio emocional, ya que las otras dos dimensiones del síndrome de desgaste profesional (despersonalización y falta de realización personal) están prácticamente ausentes en los estudiantes (32). Así, se utilizó la escala de cansancio emocional (ECE), de 10 ítems, para la que se ha notificado un coeficiente $\alpha$ de Cronbach de 0,83 (32). En un análisis de las propiedades psicométricas de esta escala en una muestra mexicana ( $n=506)$ se confirmó su estructura unifactorial y se notificó un coeficiente $\alpha$ de Cronbach de 0,90 (33).

f) Afrontamiento: uno de los instrumentos más utilizados para evaluar el afrontamiento es el cuestionario de modos de afrontamiento (Ways of Coping Questionnaire, WOCQ), de Folkman y Lazarus (21). Sin embargo, debido a indicios de falta de validez y de estabilidad de su estructura factorial, se decidió utilizar el cuestionario de afrontamiento del estrés (CAE), que evalúa siete formas de afrontamiento a través de 42 ítems, con una confiabilidad de 0,64 a 0,92 en los siete factores (34). Un análisis factorial de segundo orden evidenció dos factores que representan el afrontamiento racional y el afrontamiento focalizado en la emoción (34). g) Apoyo social percibido: basándose en una evaluación de 22 instrumentos para medir el apoyo social (35) se seleccionó el cuestionario Duke-UNC modificado y validado por Broadhead et al. (36), de 11 ítems, para el que se notificó un valor del coeficiente $\alpha$ de Cronbach de 0,90 (37).

h) Autoeficacia: se utilizó la escala de autoeficacia generalizada (38), que consta de 10 ítems. La consistencia interna de esta escala varía según los estudios psicométricos, con valores del coeficiente $\alpha$ de Cronbach comprendidos entre 0,79 y 0,93 (39).

i) Autoestima: se utilizó la escala de autoestima de Rosenberg (EAR) (40). Este cuestionario - probablemente el más utilizado para medir la autoestima- consta de diez ítems y presentó una consistencia interna satisfactoria (coeficiente $\alpha$ de Cronbach $=0,88)(41)$.

\section{Análisis estadístico}

Se estimó para cada escala la consistencia interna a través del coeficiente $\alpha$ de Cronbach. Para ajustar un modelo que pronosticara los síntomas psicosomáticos se trabajó con modelos de ecuaciones estructurales (SEM), utilizando el programa AMOS 5.0. Los modelos SEM, a diferencia de los de regresión múltiple, permiten incluir variables latentes. Las variables latentes o factores son variables no ob- 
servables que no pueden medirse directamente y se infieren a partir de variables observables, que son sus indicadores. Las variables observables, en cambio, se cuantifican a partir de medidas obtenidas por cuestionarios, encuestas, etcétera. Al modelarse las relaciones entre variables latentes, los efectos estimados no dependen del error de medida (efectos no considerados que pueden afectar la medida de una variable) (42). Además, es posible tener en cuenta las correlaciones entre los términos de error del modelo, lo que permite la inclusión de variables predictoras que se correlacionen entre sí (colinealidad). Por otro lado, los modelos SEM permiten estimar efectos de manera simultánea, de modo que una variable dependiente sea a su vez predictora de otra.

Con el primer modelo estimado se trató de confirmar el modelo formulado teóricamente (2). A partir de ese primer modelo, se identificaron los coeficientes significativos del modelo (cuyo valor es distinto de cero en la población) y se valoró la bondad del ajuste (grado de coincidencia entre la matriz de varianzas y covarianzas original, y la reproducida a partir de los coeficientes estimados). El método de estimación empleado fue el de máxima verosimilitud, comúnmente utilizado en los modelos SEM $(42,43)$. En primer lugar, se estimó la réplica del modelo inicial presentado en la figura 2. Tras la primera estimación, los valores de los coeficientes no significativos se fijaron en cero. Por último, se realizó un análisis multigrupo considerando como moderadoras las dos dimensiones del afrontamiento (racional y emocional). Los grupos de sujetos considerados en este análisis se estimaron basándose en el procedimiento de análisis factorial empleado por Sandín y Chorot respecto del CAE (34), realizando un análisis factorial exploratorio con el método de componentes principales y rotación oblimín. Se realizó luego un análisis factorial de segundo orden con las puntuaciones factoriales del primer análisis factorial exploratorio. Estos análisis se llevaron a cabo con el programa SPSS para Windows. Asimismo, se realizó un

CUADRO 2. Estadísticos descriptivos de la muestra de alumnos de la Facultad de Psicología de la Universidad Autónoma de Nuevo León, relacionados con cada variable considerada en el estudio, México, 2005

\begin{tabular}{lcrrrr}
\hline \multicolumn{1}{c}{ Variable } & $\begin{array}{c}\text { Intervalo de } \\
\text { puntuaciones } \\
\text { posibles }\end{array}$ & Mediana & Media & $\begin{array}{c}\text { Desviación } \\
\text { estándar }\end{array}$ & $\alpha^{\mathrm{a}}$ \\
\hline Síntomas psicosomáticos & $0-30$ & 6 & 6,79 & 4,07 & 0,71 \\
Estrés & $0-56$ & 21 & 21,93 & 7,03 & 0,83 \\
Depresión & $0-63$ & 5 & 6,37 & 4,98 & 0,80 \\
Ansiedad & $0-72$ & 14 & 16,33 & 11,26 & 0,93 \\
Cansancio emocional & $10-50$ & 26 & 26,47 & 8,05 & 0,89 \\
Apoyo social & $11-55$ & 48 & 46,16 & 8,18 & 0,90 \\
Autoeficacia generalizada & $10-40$ & 33 & 32,71 & 4,82 & 0,87 \\
Autoestima & $10-40$ & 36 & 35,01 & 4,37 & 0,86 \\
Afrontamiento & & & & & \\
$\quad$ Solución del problema & $0-24$ & 16 & 15,41 & 4,64 & 0,83 \\
$\quad$ Autoefocalización negativa & $0-24$ & 5 & 5,69 & 3,45 & 0,66 \\
Reevaluación positiva & $0-24$ & 16 & 16,04 & 3,68 & 0,67 \\
Expresión emocional & $0-20^{\mathrm{b}}$ & 5 & 5,75 & 3,89 & 0,86 \\
$\quad$ Evitación & $0-20^{\mathrm{b}}$ & 10 & 9,58 & 3,84 & 0,85 \\
Búsqueda de apoyo social & $0-24$ & 14 & 13,76 & 6,00 & 0,91 \\
Religión & $0-24$ & 7 & 7,42 & 5,91 & 0,87 \\
\hline
\end{tabular}

a Coeficiente $\alpha$ de Cronbach.

b Escalas en que se eliminó un ítem basándose en el análisis factorial.

análisis factorial confirmatorio mediante ecuaciones estructurales, utilizando el programa AMOS 5.0, según el método de máxima verosimilitud.

\section{RESULTADOS}

Los estadísticos descriptivos de la muestra y el grado de consistencia interna de cada escala, evaluado a través del coeficiente $\alpha$ de Cronbach, se presentan en el cuadro 2. Como puede observarse, los participantes obtuvieron puntuaciones bajas en las variables síntomas psicosomáticos, depresión y ansiedad. La media y la mediana de las puntuaciones de estrés y cansancio emocional correspondieron a la mitad de la puntuación máxima posible en cada escala. Las puntuaciones de apoyo social percibido, autoeficacia y autoestima resultaron altas. Las formas de afrontamiento con mayores puntuaciones fueron las activas.

En el primer modelo estimado (figura 3), réplica del modelo inicial (figura 2), los efectos de la ansiedad sobre los síntomas $(P=0,112)$ y del afrontamiento emocional sobre los síntomas $(P=0,053)$ no fueron significa- tivos. Los índices de bondad de ajuste y la razón entre $\chi^{2}$ y el número de grados de libertad $\left(\chi^{2} / \mathrm{gl}\right)$ indicaron que el modelo debía mejorarse (cuadro 3 ). Por lo tanto, se procedió a eliminar, uno a uno, los coeficientes no significativos.

En el modelo 2, se eliminó el efecto de la ansiedad sobre los síntomas. Tras esta modificación, todos los coeficientes resultaron significativos, sin embargo la razón $\chi^{2} / \mathrm{gl}$ y los índices de bondad de ajuste indicaron que el modelo era susceptible de ser mejorado (cuadro 3).

Por lo tanto, considerando que se pretendía detectar el modelo más parsimonioso y de mejor ajuste, se trató de mejorar el modelo eliminando los coeficientes de menor aporte para explicar los síntomas. En el modelo 3, se eliminó el efecto de la depresión sobre los síntomas, pero al hacerlo, no se observó una mejora en los índices de bondad de ajuste ni en la razón $\chi^{2} / g l$.

Considerando que la variable central está constituida por los síntomas psicosomáticos, en el modelo 4 se eliminaron las variables depresión y ansiedad, que sólo mantenían relación de dependencia con la variable estrés. Tras esta modificación, todos los coeficientes 
FIGURA 3. Modelo 1: réplica del modelo inicial para explicar el estrés y los síntomas psicosomáticos, solución estandarizada

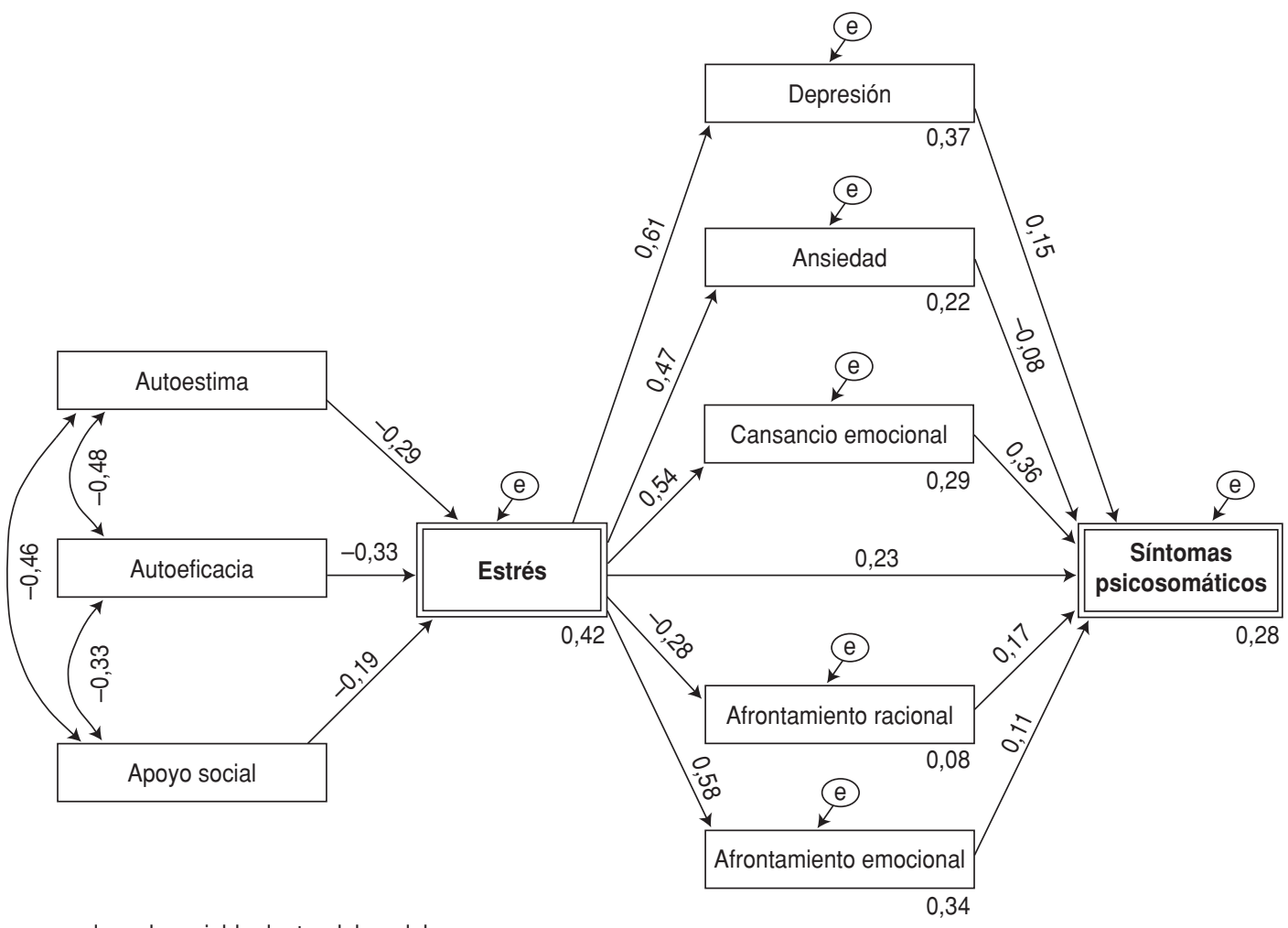

e: error de cada variable dentro del modelo.

CUADRO 3. Estadísticos de bondad de ajuste de los modelos estructurales para explicar el estrés y los síntomas psicosomáticos

\begin{tabular}{|c|c|c|c|c|c|c|c|c|c|c|c|}
\hline \multirow[b]{2}{*}{ Modelo } & \multirow[b]{2}{*}{$\chi^{2}$} & \multirow[b]{2}{*}{$\mathrm{gl}^{\mathrm{a}}$} & \multirow[b]{2}{*}{$\chi^{2} / g^{a}$} & \multicolumn{4}{|c|}{ Índices de ajuste absoluto } & \multicolumn{4}{|c|}{ Índices de ajuste incremental } \\
\hline & & & & $\mathrm{GFI}^{\mathrm{b}}$ & $A G F I^{C}$ & $\mathrm{RMR}^{\mathrm{d}}$ & RMSEA $^{\mathrm{e}}$ & $\mathrm{NFI}^{\dagger}$ & TLIg & $\mathrm{CFI}^{\mathrm{h}}$ & $\mathrm{IFI}^{\mathrm{i}}$ \\
\hline 1 & 326,031 & 28 & 11,644 & 0,850 & 0,705 & 4,003 & 0,171 & 0,767 & 0,646 & 0,780 & 0,783 \\
\hline 2 & 328,351 & 29 & 11,322 & 0,849 & 0,714 & 3,999 & 0,168 & 0,765 & 0,657 & 0,779 & 0,782 \\
\hline 3 & 334,177 & 30 & 11,139 & 0,847 & 0,719 & 4,015 & 0,167 & 0,761 & 0,663 & 0,775 & 0,778 \\
\hline 4 & 132,325 & 15 & 8,822 & 0,927 & 0,824 & 1,168 & 0,147 & 0,859 & 0,759 & 0,871 & 0,873 \\
\hline 5 & 17,213 & 6 & 2,869 & 0,985 & 0,946 & 1,394 & 0,072 & 0,973 & 0,954 & 0,982 & 0,982 \\
\hline latentes & 179,394 & 48 & 3,737 & 0,924 & 0,876 & 0,707 & 0,087 & 0,903 & 0,899 & 0,926 & 0,927 \\
\hline
\end{tabular}

a gl: grados de libertad.

b GFI: Goodness of Fit Index (índice de bondad del ajuste).

c AGFI: Adjusted Goodness of Fit Index (índice de bondad de ajuste corregido).

d RMR: Root Mean Square Residual (residuo cuadrático medio).

e RMSEA: Root Mean Square Error of Approximation (error de aproximación cuadrático medio).

f NFI: Normed Fit Index (índice de ajuste normalizado).

g TLI: Tucker-Lewis coefficient (coeficiente de Tucker y Lewis)

h CFI: Comparative Fit Index (índice de ajuste comparativo).

i IFI: Incremental Fit Index (índice de ajuste incremental).

fueron significativos; sin embargo, los estadísticos de bondad de ajuste no resultaron adecuados (cuadro 3).

Dado que ninguno de los modelos estimados presentó un ajuste acepta- ble, se estimó un modelo más parsimonioso. Teniendo en cuenta que la experiencia clínica sugiere que los síntomas suelen aparecer tras exigencias vitales que sobrepasan las capacidades perso- nales de afrontamiento (44), que altos niveles de estresores académicos predicen reacciones de estrés (45) y que el cansancio emocional es un componente del desgaste, y que se han refe- 
FIGURA 4. Modelo 5: modelo con variables observables para explicar el estrés y los síntomas psicosomáticos, solución estandarizada

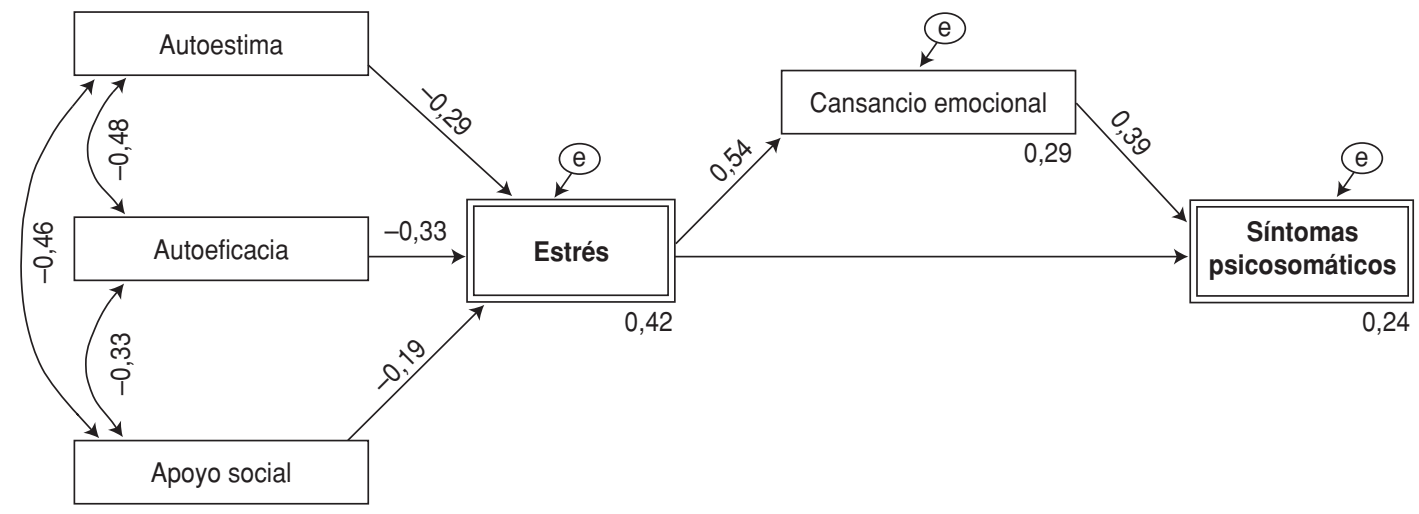

e: error de cada variable dentro del modelo.

rido trastornos psicosomáticos en personas con alto grado de desgaste (46), parece adecuada la inclusión de la variable cansancio emocional en el modelo. Por otra parte, en el modelo 1 el efecto del cansancio emocional en los síntomas fue el más alto. Por lo tanto, se valoró un quinto modelo donde el cansancio emocional y el estrés constituían variables explicativas de los síntomas (figura 4). En este modelo todos los parámetros fueron significativos; la mayoría de los índices de bondad de ajuste y la razón $\chi^{2} / g 1$ indicaron que este modelo es adecuado (cuadro 3).

Las correlaciones entre las variables autoestima, autoeficacia y apoyo social son moderadas (figura 4); estas variables explican $41,8 \%$ de la varianza del estrés. El modelo evidencia que cuanto mayor es la percepción del apoyo social, menor es el estrés percibido. Se ha postulado que la percepción del apoyo explica los efectos beneficiosos del apoyo social; la existencia de relaciones no es suficiente para observar los efectos positivos del apoyo, lo importante es la calidad de las relaciones (47). Respecto de la autoestima, además de ser esencial para que el individuo reconozca y afronte los estresores, es un recurso amortiguador del estrés (48).

El estrés explica 29,4\% de la varianza del cansancio emocional; su efecto sobre los síntomas es positivo. En el modelo, la varianza explicada de los síntomas psicosomáticos es de $24,3 \%$, valor que se considera adecuado al tomar como referencia la varianza explicada de los síntomas notificada por Orejudo y Froján (11) (16,8\% correspondía a variables psicológicas).

Se espera que quienes usan formas de afrontamiento racional tengan mejor control del estrés, menos síntomas y menos cansancio emocional; por el contrario, en quienes usan preferentemente el afrontamiento emocional, la percepción del estrés, los síntomas y el cansancio emocional parecen ser mayores. Así, se valoró este efecto moderador en un análisis de grupos múltiples respecto de las dos dimensiones del afrontamiento. Dado que al realizar el análisis factorial relativo al CAE se encontraron leves diferencias en la agrupación de ítems en cada factor; en el análisis factorial de segundo orden, la forma de afrontamiento de religión se incluyó en el factor racional, en vez de en el emocional, como en el estudio original. Esto se corroboró mediante un análisis factorial confirmatorio (49).

Los grupos de análisis se formaron basándose en las puntuaciones factoriales obtenidas para los factores de orden superior, estimando tres grupos de cada uno a partir de los percentiles (sujetos que utilizan poco, medianamente o mucho cada forma de afrontamiento).
En el análisis de grupos múltiples se trabajó con las variables desprovistas de error (puntuaciones entre las variables latentes), utilizando tres indicadores para cada factor común, para permitir así la identificabilidad del modelo $(50,51)$. Es esperable que si existe un efecto moderador del grado de afrontamiento (racional o emocional), los efectos de las variables se vean afectados, dando lugar a variaciones en la magnitud o en el signo de la estimación en cada grupo.

Al realizar los dos análisis de grupos múltiples, se llegó a las mismas conclusiones: no hay diferencia en el modelo estructural al comparar los tres grupos formados respecto del afrontamiento racional o emocional. Es posible suponer que el efecto de las variables independientes sobre las dependientes es similar en los tres grupos, aunque no puede suponerse que las varianzas-covarianzas correspondientes a las variables exógenas sean iguales; la diferencia se observa en las covarianzas no estandarizadas de autoestima, autoeficacia y apoyo social (cuadro 4). Por lo tanto, el afrontamiento no ejerce un efecto moderador de los efectos de las variables independientes sobre las dependientes.

Dado que ninguna de las variables de agrupación (afrontamiento racional o emocional) modera los efectos en el modelo estructural, en el último 
CUADRO 4. Estadísticos de bondad de ajuste del análisis multigrupo realizado considerando el afrontamiento como variable moderadora

\begin{tabular}{|c|c|c|c|c|c|c|c|c|c|}
\hline & $\chi^{2}$ & $P$ & $\mathrm{gl}^{\mathrm{a}}$ & $\chi^{2 / g l}$ & RMSEA $^{\mathrm{b}}$ & $\mathrm{CFI}$ & Dif. $\chi^{2}$ & Dif. gl & $P$ \\
\hline \multicolumn{10}{|l|}{$\begin{array}{l}\text { Variable moderadora: } \\
\text { afrontamiento racional }\end{array}$} \\
\hline \multicolumn{10}{|l|}{ Con restricciones en: } \\
\hline Pesos de medida & 284,397 & 0,001 & 156 & 1,823 & 0,048 & 0,924 & 5,775 & 12 & 0,927 \\
\hline Pesos estructurales & 301,185 & 0,001 & 168 & 1,793 & 0,047 & 0,921 & 16,789 & 12 & 0,158 \\
\hline Residuos estructurales & 341,345 & 0,001 & 186 & 1,835 & 0,048 & 0,908 & 3,875 & 6 & 0,694 \\
\hline Residuos de medida & 435,654 & 0,001 & 204 & 2,136 & 0,056 & 0,863 & 94,309 & 18 & 0,001 \\
\hline \multicolumn{10}{|c|}{$\begin{array}{l}\text { Variable moderadora: } \\
\quad \text { afrontamiento emocional }\end{array}$} \\
\hline Sin restricciones & 307,163 & 0,001 & 144 & 2,133 & 0,056 & 0,889 & $\cdots$ & $\cdots$ & $\ldots$ \\
\hline \multicolumn{10}{|l|}{ Con restricciones en: } \\
\hline Pesos de medida & 324,723 & 0,001 & 156 & 2,082 & 0,055 & 0,886 & 17,560 & 12 & 0,130 \\
\hline Pesos estructurales & 335,274 & 0,001 & 168 & 1,996 & 0,052 & 0,887 & 10,552 & 12 & 0,568 \\
\hline Residuos estructurales & 392,162 & 0,001 & 186 & 2,108 & 0,055 & 0,860 & 4,299 & 6 & 0,636 \\
\hline Residuos de medida & 513,159 & 0,001 & 204 & 2,515 & 0,065 & 0,790 & 120,997 & 18 & 0,001 \\
\hline
\end{tabular}

gl: grados de libertad.

b RMSEA: Root Mean Square Error of Approximation (error de aproximación cuadrático medio).

${ }^{c} \mathrm{CFI}$ : Comparative Fit Index (índice de ajuste comparativo).

FIGURA 5. Modelo con variables latentes para explicar el estrés y los síntomas psicosomáticos, solución estandarizada

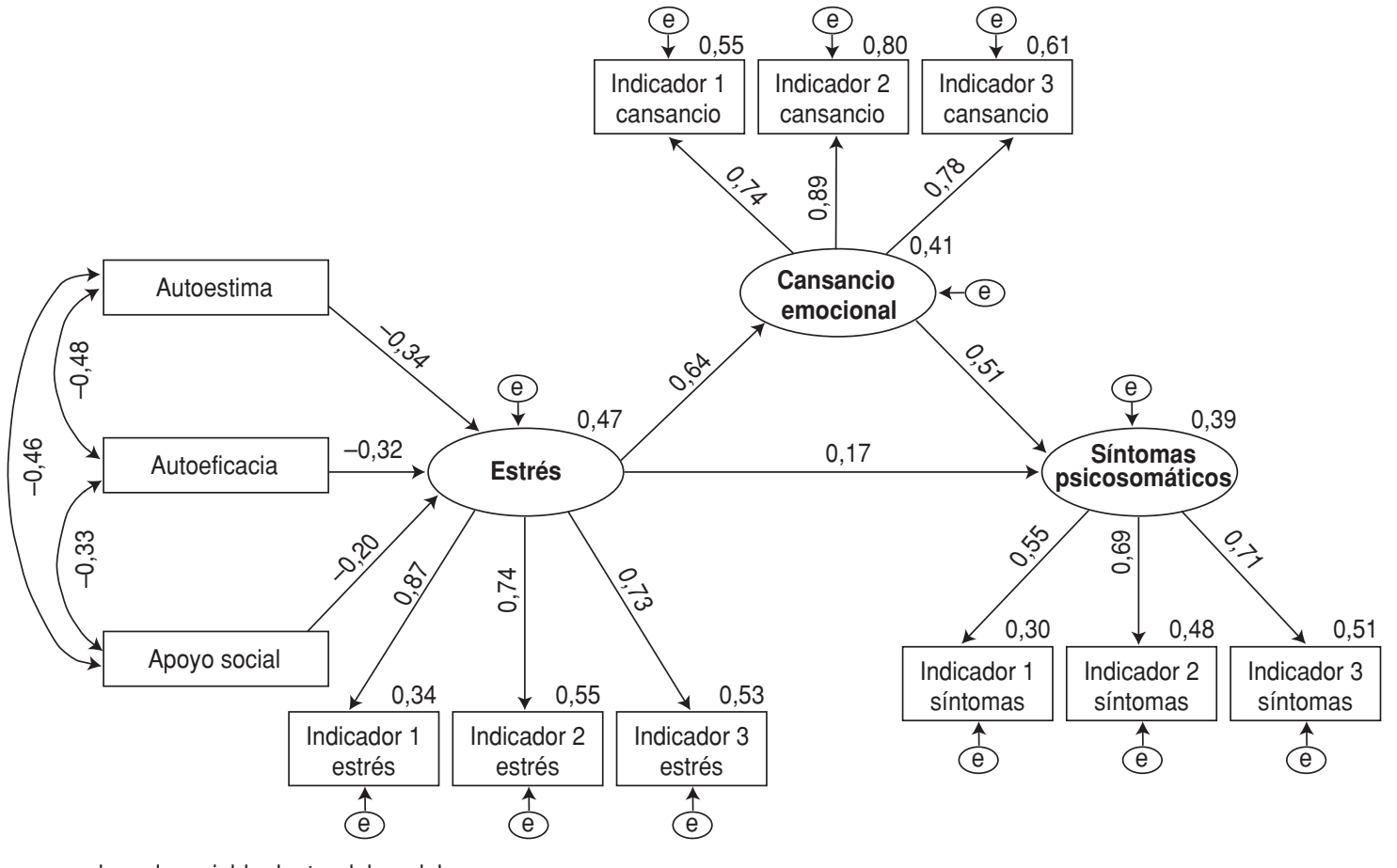

e: error de cada variable dentro del modelo.

análisis se compararon los coeficientes de los modelos con variables observables (figura 4) y con variables latentes (figura 5), tomando como referencia los intervalos de confianza de $95 \%$ (IC95\%) de los coeficientes de regresión no estandarizados, para comprobar que ambos modelos repre- sentan las mismas relaciones entre variables.

Así, los coeficientes del modelo con variables latentes relativos a las cova- 
CUADRO 5. Comparación de los coeficientes de regresión y de las varianzas estimadas entre el modelo con variables observables (figura 4) y el modelo con variables latentes (figura 5) para explicar el estrés y los síntomas psicosomáticos

\begin{tabular}{|c|c|c|c|c|c|}
\hline & \multicolumn{2}{|c|}{$\begin{array}{c}\text { Modelo con } \\
\text { variables } \\
\text { observables }\end{array}$} & \multirow{2}{*}{$\begin{array}{c}\text { IC95\%c } \\
\text { en el modelo } \\
\text { con variables } \\
\text { observables }\end{array}$} & \multicolumn{2}{|c|}{$\begin{array}{c}\text { Modelo con } \\
\text { variables } \\
\text { latentes }\end{array}$} \\
\hline & $\mathrm{NE}^{\mathrm{a}}$ & $E^{b}$ & & $\mathrm{NE}^{\mathrm{a}}$ & $E^{b}$ \\
\hline Autoestima $\Leftrightarrow$ Autoeficacia & 10,07 & 0,48 & $7,68-12,46$ & 10,07 & 0,48 \\
\hline Autoestima $\Leftrightarrow$ Apoyo social & 16,29 & 0,46 & $12,27-20,32$ & 16,29 & 0,46 \\
\hline Autoeficacia $\Leftrightarrow$ Apoyo social & 13,10 & 0,33 & $8,84-17,36$ & 13,10 & 0,33 \\
\hline Estrés $\Leftarrow$ Autoestima & $-0,47$ & $-0,29$ & $-0,63--0,32$ & $-0,21$ & $-0,34$ \\
\hline Estrés $\Leftarrow$ Autoeficacia & $-0,48$ & $-0,33$ & $-0,61--0,35$ & $-0,18$ & $-0,32$ \\
\hline Estrés $\Leftarrow$ Apoyo social & $-0,16$ & $-0,19$ & $-0,24--0,09$ & $-0,07$ & $-0,20$ \\
\hline Cansancio emocional $\Leftarrow$ Estrés & 0,62 & 0,54 & $0,52-0,72$ & 0,78 & 0,64 \\
\hline Síntomas psicosomáticos $\Leftarrow$ Estrés & 0,09 & 0,16 & $0,03-0,15$ & 0,08 & 0,17 \\
\hline Síntomas psicosomáticos $\Leftarrow$ Cansancio emocional & 0,20 & 0,39 & $0,14-0,25$ & 0,19 & 0,51 \\
\hline Varianza de los síntomas psicosomáticos (\%) & \multicolumn{2}{|c|}{24,30} & $\ldots$ & \multicolumn{2}{|c|}{39,40} \\
\hline Varianza del cansancio emocional (\%) & \multicolumn{2}{|c|}{29,40} & $\ldots$ & \multicolumn{2}{|c|}{40,90} \\
\hline Varianza del estrés (\%) & \multicolumn{2}{|c|}{41,80} & $\ldots$ & \multicolumn{2}{|c|}{47,50} \\
\hline
\end{tabular}

$\mathrm{NE}$ : coeficiente de regresión no estandarizado.

${ }^{b} \mathrm{E}$ : coeficiente de regresión estandarizado.

c IC95\%: intervalo de confianza de $95 \%$ de los coeficientes no estandarizados.

rianzas entre la autoestima y la autoeficacia, la autoestima y el apoyo social, la autoeficacia y el apoyo social, y el efecto del estrés sobre los síntomas psicosomáticos están comprendidos dentro de los IC95\% de los coeficientes del modelo con variables observables. Por lo tanto, se puede afirmar que en estos casos no han cambiado las estimaciones y, por consiguiente, los efectos de unas variables sobre otras. Sólo en los casos del efecto de las variables exógenas sobre el estrés y del estrés sobre el cansancio emocional los valores quedan fuera de los IC95\%, conservándose la dirección en cada una de las relaciones planteadas (cuadro 5). El efecto del estrés sobre los síntomas es directo e indirecto; en el modelo 5, el valor estandarizado relativo a los efectos totales es de $0,369(0,158$ respecto del efecto directo y 0,211 respecto del indirecto), mientras que en el modelo con variables latentes, el valor estandarizado relativo a los efectos totales es de $0,491(0,167$ respecto del efecto directo y 0,324 respecto del indirecto).

\section{CONCLUSIONES}

En este estudio se realizó un análisis parcial del modelo teórico planteado anteriormente (2), dejando para estudios posteriores la valoración de la si- tuación por parte del individuo, así como la evaluación de los estresores para la población objetivo. Los resultados obtenidos no confirmaron totalmente el modelo inicial, formado por 10 variables (figura 2). Se considera que la complejidad del modelo influyó para que no todos los coeficientes resultaran significativos.

El modelo presentado constituye el principal aporte de este trabajo. Se trata de un modelo explicativo de síntomas psicosomáticos, con buen ajuste, que explica $24,3 \%$ de la varianza al utilizar variables observables y $39,4 \%$ al utilizar variables latentes.

El modelo se analizó considerando las variables como observables y como latentes con sus respectivos indicadores (grupos de ítems de las escalas utilizadas). Así, se encontró que el porcentaje de varianza explicada aumenta al utilizar variables desprovistas de error.

Basándose en los modelos de ecuaciones estructurales elaborados, se confirma que el apoyo social percibido, la autoeficacia y la autoestima son predictores del estrés, el estrés es predictor del cansancio emocional y de los síntomas psicosomáticos, el cansancio emocional es predictor de los síntomas y el estrés influye de manera importante en los síntomas, ya que tiene efectos directo e indirecto sobre ellos.
Al comparar las relaciones encontradas en el modelo con los planteamientos de la teoría transaccional y del modelo procesual, se confirma que la valoración que hace el sujeto de sus recursos influye en el grado de estrés percibido y sus consecuencias (cansancio emocional y síntomas psicosomáticos). Asimismo, tal como menciona Sandín (19), se confirma que hay diversos componentes relevantes relacionados con el estrés.

Con las variables medidas en el estudio no es posible contrastar la influencia que tiene en el estrés percibido la valoración de una situación específica como amenazante o de daño o perdida, dado que no se evaluaron estresores. Así, no se puede confirmar que la valoración de la situación por parte del sujeto repercute en el grado de estrés percibido.

Por otra parte, a pesar de que el afrontamiento es un elemento central de la teoría transaccional del estrés y del modelo procesual de Sandín, los resultados de los análisis de grupos múltiples ponen en duda el efecto moderador de las formas de afrontamiento sobre el estrés y sus consecuencias. Estos resultados nos hacen dirigir la atención a las primeras fases del proceso de estrés, es decir, trabajar con los sujetos desde una perspectiva de prevención y tratamiento del estrés, 
contemplando la evaluación cognitiva de los eventos y los recursos propios, antes de centrar los esfuerzos en modificar las formas de afrontamiento.

Una aplicación inmediata de este trabajo es la utilización del modelo presentado para proporcionar a los pacientes y los profesionales de la salud una explicación científica de los síntomas psicosomáticos, enmarcada en una de las teorías del estrés más difundidas y aceptadas, la teoría transaccional. Utilizando este modelo, se puede explicar a los pacientes que los síntomas psicosomáticos son el producto de una combinación de variables, y no el resultado exclusivo de algún evento externo. Con el modelo como marco para explicar el estrés se puede resaltar que cada persona es parte activa de su estrés (según la teoría transaccional, al cambiar la percepción que se tiene de alguna situación o de sus recursos, se modifica la percepción del estrés).

En el trabajo terapéutico individual resultaría inadecuado centrarse en una parte del proceso de estrés, puesto que deben considerarse todos los elementos involucrados (11). Sin embargo, en un trabajo grupal, es necesario implementar lo que puede funcionar en la mayoría de las personas. Así, se propone que en los modelos de intervención para la prevención y el tratamiento de síntomas psicosomáticos se trabajen estrategias para mejorar el apoyo social, la autoeficacia y la autoestima, puesto que estas variables explican un porcentaje importante del estrés percibido $(41,8 \%$ en el modelo con variables observables y $47,5 \%$ en el modelo con variables latentes) y pueden funcionar como protectores de las consecuencias del estrés. También es aconsejable utilizar técnicas de reestructuración cognitiva para cambiar la valoración de la situación estresante, cuando se interprete falsamente como desbordante de los recursos del indivi- duo. Se recomienda evitar intervenciones centradas exclusivamente en el manejo del estrés y el cansancio emocional, en el caso de los estudiantes, y del desgaste, en otras poblaciones.

En investigaciones centradas en los síntomas psicosomáticos, el empleo del modelo de este estudio como marco de referencia puede aportar las siguientes ventajas: a) en trabajos experimentales, enfocados hacia estrategias de prevención o tratamiento, este modelo permite contemplar variables que explican un importante porcentaje de varianza de los síntomas; b) en investigaciones sin intervención, destinadas a estudiar variables relacionadas con los síntomas psicosomáticos, este modelo constituye un punto de partida.

Es preciso tener en cuenta que los síntomas son de origen multifactorial y que los predictores incluidos en el modelo presentado corresponden solamente a variables psicosociales. Así, es importante valorar la inclusión de variables tales como aspectos de personalidad, factores ambientales y antecedentes médicos. $\mathrm{Al}$ incluir aspectos de personalidad, como el neuroticismo, el afecto negativo y el optimismo, se recomienda utilizar modelos de grupos múltiples, como los empleados en el presente estudio para evaluar el efecto moderador del afrontamiento.

Para continuar este estudio, será necesario incluir en el modelo explicativo los estresores relevantes para la población objetivo, así como la evaluación que el individuo hace de ellos. Como se ha mencionado, la valoración de los estímulos estresantes representa un aspecto central al contrastar la teoría transaccional del estrés. Al tomar como base el modelo de este estudio, se elimina una de las dificultades afrontadas al inicio de la investigación: la selección de las variables que se medirán para su inclusión en el modelo estructural. Esta dificultad está supe- rada en el modelo simplificado (figuras 4 y 5), lo que permite, en futuras investigaciones, el estudio de otras variables, como los estresores, sin que esto plantee problemas en la evaluación (por la extensión del instrumento empleado para medir las variables).

Al evaluar estresores, deben contemplarse las situaciones particulares de cada grupo (por ejemplo: estresores de estudiantes, profesionales de la salud, profesores, cuidadores de personas con enfermedades crónicas, etcétera), incluyendo los eventos más significativos para cada población.

Asimismo, es necesario continuar las investigaciones con universitarios, ya que el grado de estrés detectado en esta población es cercano al detectado en muestras clínicas (personas con enfermedades crónicas) evaluadas con el mismo cuestionario (26). Los universitarios son una población particular, que está expuesta a estresores y a sus efectos, y no sólo un grupo de estudio fácilmente accesibles. Es importante estudiar los efectos del cansancio emocional, el estrés y los síntomas psicosomáticos en la formación profesional y el rendimiento académico de los estudiantes universitarios. Posteriormente, es necesario que se contemplen la prevención y el tratamiento del estrés, el cansancio emocional y los síntomas en los estudiantes.

Por último, es preciso evaluar este modelo explicativo de síntomas psicosomáticos con muestras que presenten mayor cantidad de síntomas y que estén expuestas a más estresores que los estudiantes - ya que el trabajo con una población sana tiene la limitación de que las relaciones entre las variables no se manifiestan con suficiente magnitud-, incluyendo nuevamente el análisis del posible efecto moderador de afrontamiento y utilizando medidas específicas del desgaste en la población objetivo.

\section{REFERENCIAS}

1. López J, Belloch A. La somatización como síntoma y como síndrome: una revisión del trastorno de somatización. Rev Psicopatol Psicol Clin. 2002;7(2):73-93.
2. González MT, Landero R. Síntomas psicosomáticos y teoría transaccional del estrés. Ansiedad Estrés. 2006;12(1):45-61.
3. Sandín B, Chorot P, Santed M, Jiménez M. Trastornos psicosomáticos. En: Belloch A, Sandín B, Ramos F. eds. Manual de psicopa- 
tología, Vol. 2. Madrid: McGraw-Hill; 1995 Pp. 402-69.

4. Gureje O, Simon G, Ustun T, Goldberg D. Somatization in cross-culural perspective: A World Health Organization study in Primary Care. Am J Psychiatry. 1997;154(7):989-95.

5. Kirmayer L, Groleau D, Looper K, Dominicé M. Explanining medically unexplained symptoms. Can J Psychiatry. 2004;49(10):663-71.

6. Lieb R, Zimmermann P, Friss R, Hofler M Tholen S, Wittchen $H$. The natural course of DSM-IV somatoform disorders and syndromes among adolescents and young adults: a prospective-longitudinal community study. Eur Psychiatry. 2002;17(6):321-31.

7. Natvig G, Albrektsen G, Aderssen N, Qvarnstrom U. School-related stress and psychosomatic symptoms among school adolescents. J Sch Health. 1999;69(9):362-8.

8. González MT, Landero R. Síntomas psicosomáticos y estrés en estudiantes de Psicología. Rev Psicol Soc. 2006;21(2):141-52.

9. Lipowski Z. Somatization: the concept and its clinical approach to the somatizing patient. Am J Psychiatry. 1988;145(11):1358-68.

10. Matud M, Bethencourt J. Ansiedad, depresión y síntomas psicosomáticos en una muestra de amas de casa. Rev Latinoam Psicol. 2000; 32(1):91-106.

11. Orejudo S, Froján M. Síntomas somáticos: Predicción diferencial a través de variables psicológicas, sociodemográficas, estilos de vida y enfermedades. An Psicol. 2005;21(2):276-85.

12. Lipsane T, Saarijävi S, Lauerma H. Exploring the Relations between Depression, Somatization, Dissociation and AlexithymiaOverlapping or Independent Constructs? Psychopathol. 2004;37(4):2006.

13. Kooiman C, Bolk J, Brand R, Trijsburg R, Rooijmans $\mathrm{H}$. Is alexithymia a risk factor for unexplained physical symptoms in general medical outpatients? Psychosom Med. 2000; 62:768-78.

14. Fernández R, Fernández C. Actitudes hipocondríacas, síntomas somáticos y afecto negativo. Psicothema. 1998;10(2):259-70.

15. Santed M, Sandín B, Chorot P, Olmedo M, García-Campayo J. The role of negative and positive affectivity on perceived stresssubjective health relationships. Acta Neuropsychiatr. 2003;15:199-216.

16. Cronkite R, Moos R. The role of predisposing and moderating factors in the stress-illness relationship. J Health Soc Behav. 1984;25(4): 372-93.

17. Holloway KL, Zerbe KJ. Simplified approach to somatization disorder: when less may prove to be more. Postgrad Med. 2000;108(6): 89-95.

18. Lazarus R, Folkman S. Stress, coping and adaptation. New York: Springer; 1984.

19. Sandín B. Estrés psicosocial. Madrid: Klinik; 1999.

20. Sandín B. El estrés. En: Belloch A, Sandín B y Ramos F. eds. Manual de psicopatología, Vol. 2. Madrid: McGraw-Hill; 1995. Pp. 3-52.

21. Olmedo E, Ibáñez I, Hernández L. Validación estructural del Ways of coping questionnaire de Lazarus y Folkman: un análisis factorial restrictivo. Ansiedad Estrés. 2001;7(1):15-28.

22. Fernández J, Rusiñol J. Economía y psicología: costes por el bajo rendimiento académico relacionado con la ansiedad ante los exámenes y las pruebas de evaluación. [Sitio en Internet] Universitat Oberta de Catalunya. Hallado en http://www.uoc.edu/dt/20128/. Acceso el 1 diciembre 2004.

23. Kroenke K, Spitzer RL, Williams J. The PHQ15: Validity of a new measure for evaluating the severity of somatic symptoms. Psychosom Med. 2002;64:258-66.

24. Interian A, Allen L, Gara M, Escobar J, DíazMartínez A. Somatic complaints in primary care: further examining the validity of the Patient Health Questionnaire (PHQ-15). Psychosomatics. 2002;47(5):392-98.

25. Cohen S, Kamarak T, Mermelstein R. A Global Measure of Perceived Stress. J Health Soc Behav. 1983;24:385-96.

26. González MT, Landero R. Factor Structure of the Perceived Stress Scale (PSS) in a Sample from Mexico. Span J Psychol. 2007;10(1): 199-206.

27. Lazarus R, DeLongis A, Folkman S, Gruñe R. Stress and adaptational outcomes: The problem of confounded measures. Am Psychol. 1985;40(7):770-9.

28. Beck A, Steer R, Garbin M. Psychometric properties of the Beck Depression Inventory. Twenty-five years of evaluation. Clin Psychol Rev. 1988;8:77-100.

29. Inglés C, Méndez X, Hidalgo M, Rosa A, Orgilés $M$. Cuestionarios, inventarios y escalas de ansiedad social para adolescentes: una revisión crítica. Rev Psicopatol Psicol Clin. 2003;8(1):1-21.

30. La Greca AM, López N. Social anxiety among adolescents: Linkages with peer relations and friendships. J Abnorm Child Psychol. 1998; 26(2):83-94.

31. Olivares J, Ruiz J, Hidalgo M, García-López L, Rosa A, Piqueras J. Social anxiety scale for adolescents (SAS-A): psychometric properties in a Spanish-speaking population. Int J Clin Health Psychol. 2005;5(1):85-97.

32. Ramos F, Manga D, Moran C. Escala de cansancio emocional (ECE) para estudiantes universitarios: propiedades psicométricas y asociación. Interpsiquis [Publicación periódica en línea] 2005. [citada abril 22]. Hallado en: http://www.psiquiatria.com/articulos/ estres/20478/

33. González MT, Landero R. Escala de cansancio emocional (ECE) para estudiantes universitarios: propiedades psicométricas en una muestra de México. An Psicol. En preparación, 2007.

34. Sandín B, Chorot P. Cuestionario de afrontamiento del estrés (CAE): desarrollo y validación preliminar. Rev Psicopatol Psicol Clin 2003;8(1):39-54.

35. Terol M, López S, Neipp M, Rodríguez J, Pastor M, Martín-Aragón M. Apoyo social e instrumentos de evaluación: revisión y clasificación. Anuario Psicol. 2004;35(1):23-45.

36. Broadhead WE, Gehlbach SH, Degruy FV, Kaplan BH. The Duke-UNC functional social support questionnaire: measurement of social support in family medicine patients. Med Care. 1998;26:709-23.

37. Bellón J, Delgado A, Luna J, Lardelli P. Validez y fiabilidad del cuestionario de apoyo social funcional Duke-UNC-11. Aten Primaria. 1996;18(4):153-63.

38. Jerusalem M, Schwarzer R. Self-efficacy as a resource factor in stress appraisal processes. En Schwarzer R. ed. Self-efficacy: thought control of action Washington, D.C.: Hemisphere; 1992. Pp. 195-213.

39. Scholz U, Gutiérrez-Doña B, Sud S, Schwarzer $R$. Is general self-efficacy a universal construct? Psychometric findings from 25 countries. Eur J Psychol Assess. 2002;18(3):242-51.

40. Rosenberg M. Society and the Adolescent Self-Image. Edición revisada. Middletown, CT: Wesleyan University Press; 1989.

41. Zimprich D, Perren S, Hornung R. A twolevel confirmatory factor analysis of a modified Rosenberg Self-esteem scale. Educ Psychol Meas. 2005;65(3):465-81.

42. Ruiz M. Introducción a los modelos de ecuaciones estructurales. Madrid: Ediciones UNED; 2000.

43. Schermelleh-Engel K, Moosbrugger H, Müller H. Evaluating the fit of Structural Equation Models: Tests of significance and descriptive goodness-of-fit measures. Methods of psychological research online. 2003;8(2):23-74

44. Farré F, Fullana M. Recomendaciones terapéuticas en terapia cognitivo-conductual. España: Ars Médica; 2005.

45. Misra R, Crist M, Burant C. Relationships Among Life Stress, Social Support, Academic Stressors, and Reactions to Stressors of International Students in the United States. Int J Stress Manag. 2003;10(2):137-57.

46. Fernández G. Burnout y trastornos psicosomáticos. Revista de psicología del trabajo y de las organizaciones. 2000;16(2):229-35.

47. Musitu G, Buelga S, Lila MS, Cava MJ. Familia y adolescencia: análisis de un modelo de intervención psicosocial. Madrid: Síntesis; 2001.

48. Lila M, Buelga S, Musitu G. Las relaciones entre padres e hijos en la adolescencia. Madrid: Pirámide; 2006.

49. González MT, Landero R. Cuestionario de Afrontamiento del Estrés (CAE): Validación en una muestra mexicana. Rev Psicopatol Psicol Clin. En preparación, 2007.

50. Bollen KA. A new incremental fit index for general structural equation models. Sociol Methods. Res 1989;17:303-16.

51. Fabrigar L, MacCallum R, Wegener DT, Strahan EJ. Evaluating the use of Exploratory Factor Analysis in Psychological Research. Psychol Methods. 1999;4(3):272-99.

Manuscrito recibido el 27 de diciembre de 2006. Aceptado para publicación, tras revisión, el 2 de agosto de 2007. 
ABSTRACT Objective. To evaluate a descriptive model of stress that integrates an explanation of psychosomatic symptoms with the transactional theory of stress.

\section{Confirmation of a descriptive model of stress and psychosomatic symptoms using structural equations} Methods. A random and stratified representative sample was selected from among the students in the School of Psychology at the Autonomous University of Nuevo León, Mexico. The subjects were chosen randomly from a student directory provided by the school. A self-administered questionnaire was used that contained the psychometric properties necessary for accurately quantifying each of the variables included in the model. Results were analyzed using AMOS 5.0, employing the best probability method. Also, the structural model was compared using discrete variables only and endogenous latent variables.

Results. The results that were obtained partly confirmed the model and corroborated the impact that stress and emotional exhaustion have on psychosomatic symptoms and that self-esteem, self-efficacy, and social support have on stress. The model with discrete variables [chi square test/degrees of freedom $\left(\chi^{2} / \mathrm{df}\right)=2.87$; goodness of fit $(\mathrm{GFI})=0.985$; adjusted goodness of fit $(\mathrm{AGFI})=0.946$; root mean square error of approximation (RMSEA) $=0.072$; incremental fit index (IFI) $=0.982]$ has a better fit than the model with latent variables $\left(\chi^{2} / \mathrm{df}=3.74 ; \mathrm{GFI}=0.924 ; \mathrm{AGFI}=0.876\right.$; RMSEA $=0.09$, IFI $=0.927)$. In both cases, the fit is adequate.

Conclusions. The model discussed is the main contribution of this study. It is a descriptive model of psychosomatic symptoms, with a good fit, that describes $24.3 \%$ of the variance for discrete variables and $39.4 \%$ when using latent variables.

Key words Psychophysiologic disorders, stress, Mexico. 